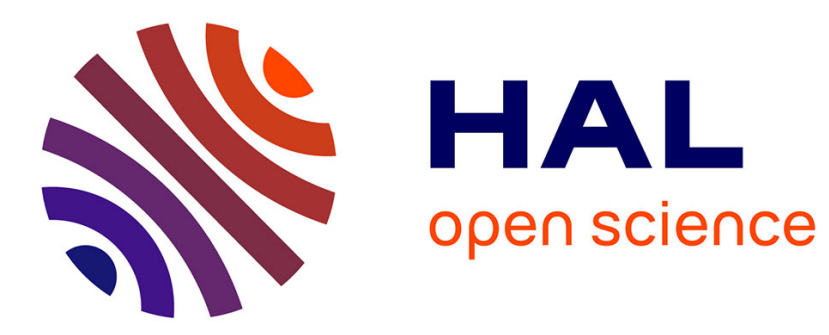

\title{
Malaise dans l'évaluation
}

Paul-Laurent Assoun

\section{- To cite this version:}

Paul-Laurent Assoun. Malaise dans l'évaluation. Journal français de psychiatrie, 2007, 29, pp.11 - 16. 10.3917/jfp.029.0011 . hal-01501926

\section{HAL Id: hal-01501926 https://hal.science/hal-01501926}

Submitted on 4 Apr 2017

HAL is a multi-disciplinary open access archive for the deposit and dissemination of scientific research documents, whether they are published or not. The documents may come from teaching and research institutions in France or abroad, or from public or private research centers.
L'archive ouverte pluridisciplinaire HAL, est destinée au dépôt et à la diffusion de documents scientifiques de niveau recherche, publiés ou non, émanant des établissements d'enseignement et de recherche français ou étrangers, des laboratoires publics ou privés. 


\section{MALAISE DANS L'ÉVALUATION}

Paul-Laurent Assoun

\section{ERES | «Journal français de psychiatrie »}

2007/2 n²9| pages 11 à 16

ISSN 1260-5999

ISBN 9782749206240

Article disponible en ligne à l'adresse :

http://www.cairn.info/revue-journal-francais-de-psychiatrie-2007-2-page-11.htm

\section{Pour citer cet article :}

Paul-Laurent Assoun, " Malaise dans l'évaluation », Journal français de psychiatrie 2007/2 ( $\left.\mathrm{n}^{\circ} 29\right)$, p. 11-16.

DOI $10.3917 /$ jfp.029.0011

Distribution électronique Cairn.info pour ERES.

(C) ERES. Tous droits réservés pour tous pays.

La reproduction ou représentation de cet article, notamment par photocopie, n'est autorisée que dans les limites des conditions générales d'utilisation du site ou, le cas échéant, des conditions générales de la licence souscrite par votre établissement. Toute autre reproduction ou représentation, en tout ou partie, sous quelque forme et de quelque manière que ce soit, est interdite sauf accord préalable et écrit de l'éditeur, en dehors des cas prévus par la législation en vigueur en France. Il est précisé que son stockage dans une base de données est également interdit. 


\title{
Malaise dans l'évaluation
}

\author{
Paul-Laurent Assoun*
}

"On ne peut se défendre de l'impression que les hommes mesurent communément avec de faux critères. »

S. Freud, Malaise dans la civilisation 1

L a phrase liminaire de l'essai où le créateur de la psychanalyse saisit le malaise au cour de la constat d'une faillite générale de l'évaluation comme fait anthropologique. Elle énonce tranquillement que l'homme se trompe très généralement sur son évaluation de lui-même. En d'autres termes, une observation impartiale et lucide atteste que le genre humain vise foncièrement « à côté de la plaque $» .$.

On n'a pas assez souligné que le diagnostic freudien sur le malaise collectif commence par un petit traité critique du bonheur qui repose sur ce diagnostic d'une autotromperie dans l'évaluation du « bien » et des « biens ». Freud prend acte que les hommes tendent généralement vers « pouvoir, succès et richesse » et qu'ils n'affectent admirer chez les autres les « vraies valeurs de la vie » que pour les sous-estimer systématiquement dans leur agir propre.

L'erreur ou la faute dans l'évaluation - on trouve ici les mots Massstäben (qui désigne les « lignes directrices » ou échelles d'évaluation, bref les critères) et Werte (qui désigne les valeurs) - initialise en quelque sorte l'éthique freudienne, articulée à son expérience anthropologique incomparable, acquise depuis le réel inconscient. Façon de dire

* Professeur à l'Université Paris-7-Diderot, psychanalyste, membre d' « Espace analytique ». que « l'humain » s'évalue foncièrement mal - ce qui introduit un « tordu axial » au cœur du fait anthropologique. Tel est « l'humain » qu'il est enclin à sous-estimer le meilleur et à sur-estimer le pire. Cela n'ouvre chez Freud nulle déploration ou lamentation, mais organise une clinique de l'illusion des plus lucides - sauf à reconnaître la « bigarrure » des attitudes qui s'organisent sur cette toile de fond de tromperie.

\section{La raison évaluative}

Or, par une ironie d'emblée révélatrice, on sait que c'est sous la forme de l'évaluation que s'exerce désormais de façon prévalente la dévaluation de la psychanalyse. Il y a là l'ambition d'évaluer correctement et objectivement. Après tout, que signifie cette interpellation par l'évaluation, plus spécialement de ce que l'on désigne comme «psychothérapies »?

Celle-ci est certes présentée comme une pure et simple exigence technique. Façon de «noter » une prestation au moyen de paramètres. Mais le terme lui-même est lourd d'une connotation axiologique : pas d'évaluation sans « système de valeurs ", que l' « évaluateur» les exhibe ou non. De quelle « valuation » s'agit-il ?

Que s'agit-il d'évaluer ici ? Que veut l'évaluateur? La réponse la plus immédiate est qu'il veut améliorer
L'homme se

trompe très

généralement

sur son

évaluation

de lui-même.

1. S. Freud, Malaise dans la civilisation, G.W.XIV, 421. (Nous citons les textes de Freud d'après les Gesammelte Werke, Fischer Verlag en retraduisant les passages concernés.) l'action, mais il en ressort que cela revient à dévaluer l'acte, électivement pointé dans l'acte analytique. $\mathrm{Si}$ même ce n'en était pas l'intention, c'en est à coup sûr l'effet. Le procès d'intention n'est pas de mise, dès lors que l'intention est largement affichée et rationalisée : celle du bilan initial, de la définition de l'objectif, de la mise en programme et de l'évaluation terminale des prestations.

Pourtant ce constat vaut comme conclusion d'une enquête qui reste à mener. On évoquera immédiatement la volonté sociale de paramétrer les actions thérapeutiques pour les ajuster, comme toute réalité, au coût social. Vaste opération d'économie et de « mélioration ». Reste à comprendre ce que doit être cette «mentalité de l'évaluation » pour viser comme effet, sinon finalité, la dévaluation de la praxis.

Partons d'un constat empirique : là où il $\mathrm{y}$ a prestation, il y a performance. Et toute performance est virtuellement quantifiable. Cela donne raison à qui demande l'évaluation. Mais voilà le point à rappeler : Freud n'a jamais dit autre chose. Il y a bien chez le créateur de la psychanalyse un mouvement réflexif sur l'acte, mais qui ne passe pas par la ratio quantitative.

Certes, l'auteur de la science des rêves a soin de rappeler qu'il a déchiffré des milliers de rêves avant d'avan- 
cer son axiomatique onirique du désir2. Il lui arrive aussi de noter le nombre de patients sur lesquels s'appuie tel déchiffrement de fantasme ${ }^{3}$, de noter la limite d'exemplaires de comportement masochiste 4 ou de mélancolie 5 - notations souvent méconnues par les lecteurs analystes eux-mêmes qui lui donnent trop hâtivement valeur de structure. Mais celle-ci vient prendre à rebours l'évaluationnisme actuel. «Le fait isolé l'attirait et il ne pouvait le chasser de son esprit qu'après l'avoir expliqué », dit Jones à propos... « C'est ainsi que travaillait la pensée de Freud. Chaque fois qu'il lui arrivait d'observer un fait simple mais significatif, il sentait et savait qu'il y avait là quelque chose de général ou d'universel »- alors même que " l'idée d'établir sur ce point des statistiques lui était tout à fait étrangère 6 ».

\section{L'impératif d'évaluation}

Le comble de "l'objectivité » évaluationniste, c'est qu'elle enjoint à ses adversaires de s'auto-évaluer. Elle les crédite de cette prérogative de s'appliquer, comme agents, ces critères supposés objectifs de l'action, donc applicables à quiconque et à chacun.

Nous sommes dans un modèle « machinique » de traitement de l'information. Il s'agit de mesurer ce qui entre et sort de la « boîte noire » et la différence entre deux « états » de la prestation machinique.

Ce modèle promeut un véritable style surmoïque en trois temps logiques : "évaluons-nous », " évaluez-vous », " évaluons... vous ». L'évaluateur se veut non partisan, puisque prêt à s'incliner devant la loi de l'évaluation - sauf à ce que, au nom de cet universel pragmatique, c'est l'acte même que l'on veut mortifier. En fait de loi, nous sommes plutôt dans une logique de la réglementation et du règlement, que nous avons située ailleurs 7 .

Cela pose la question de « l'efficace » de l'analyse 8 dont il faudra rappeler les attendus jusqu'à ses incidences sur l'idée de guérison 9 , afin d'y situer la problématique de l'évaluation et ses enjeux.

\section{Évaluer : du mot à la chose. Valeur et calcul}

Prenons d'abord la mesure du mot qui contribue à formater la chose pour en soutenir l'ambiguiité.

L'évaluation désigne l'acte d'évaluer. Et évaluer, c'est, nous dit le lexicographe, « porter un jugement sur la

\section{Il s'agit de}

mesurer ce qui

entre et sort de

la «boîte

noire » et la

différence entre

\section{deux «états»}

de la prestation

machinique.

2. S. Freud, L'interprétation des rêves.

3. S. Freud, Un enfant est battu. 4. S. Freud, Le problème économique du masochisme.

5. S. Freud, Deuil et mélancolie. 6. E. Jones, La vie et l'œuvre de Sigmund Freud, Paris, PUF, t. I, p. 106-107 et notre commentaire dans Introduction à

l'épistémologie freudienne, Paris, Payot, 1981-1990 p. 111-112. 7. P.-L. Assoun, Le préjudice et l'idéal. Pour une clinique sociale du trauma, Anthropos/Economica, 1999, p. 211-234.

8. P.-L. Assoun, « Du métier impossible à l'acte nécessaire. L'effet analytique », dans Figures de la psychanalyse, $\mathrm{n}^{\circ} 15$, éditions érès, p. 17-31, 2007.

9. P.-L. Assoun, « La guérison à l'épreuve de la psychanalyse ", dans Synapse, 2007. 10. P.-L. Assoun, Marx et la répétition historique, Presses universitaires de France, coll. « Quadrige », 2e éd., 1998.

11. Émile Goblot, La logique des jugements de valeur.
Il y a donc d'une part un registre qualitatif, de l'autre une économie du coût.

D'une part, l'évaluateur - instance à situer - manie une « pierre de touche » ou « étalon » applicable à quiconque pour mesurer la réalité empirique ainsi jaugée et par là même jugée de facto.

D'autre part, il cherche à déterminer une quantité par le calcul, avec cette nuance déterminante que cette « calculation » se fait sans recourir à la mesure directe, dans les sciences dites de l'homme.

Évaluer, c'est donc à la fois indexer une réalité à une grille de valeurs et produire un calcul pour ainsi dire. Nous sommes donc face à une axiologie utilitariste.

La valeur, c'est le caractère mesurable d'un objet, en tant que susceptible d'être échangé - quoique l'on parle a contrario d'une « valeur d'usage »- dichotomie dont Marx, en « clinicien du social 10 », fera un usage majeur. Par extension métaphysique, la valeur en vient à définir le caractère de ce qui obéit à une norme idéale ou à un type - détermination axiologique qui ouvre une logique du jugement 11 .

L'évaluation se déplace donc sans complexes de l'idéalisme normatif aux calculs de boutique et de boutiquier.

La raison évaluative se déploie entre ces deux pôles du jugement dit de valeur et de la mesure, intendance d'épicerie ou logique du « bocal ». Il faut nous-même en prendre la mesure pour situer sa signification dans la condition contemporaine et le « malaise de la culture » correspondant.

\section{L'effet attendu}

Qu'est-ce qui est donc à évaluer quand il s'agit d'un acte? D'un mot, son effet. Repassons donc par un examen de ce registre de l'efficacy. Est dit « efficace » ce « qui produit l'effet qu'on en attend »-qu'il s'agisse d'un traitement ou d'un médicament, ou encore d'une aide, dans la mesure où ils ont engendré l'effet escompté. Nous sommes dans une logique de l'action.

On parlera d' « efficacité » pour désigner la «capacité de produire le maximum de résultats avec le minimum d'effort et de dépense »-ce qui renvoie à la notion de rendement. Il y va en effet du produit effectif d'un travail, voire de sa «plus-value » (notion originairement agricole : production de la terre, évaluée par rapport à l'unité de surface cultivée). Cela pose enfin la question de l'efficience, qui met l'accent sur l'efficacité en acte. 
Il n'y a de là qu'un pas à conclure sur... l'évaluation. Car il est logique, il est même exigible, dès lors que l'on a accrédité cette notion d'un effet attendu, donc programmable, que l'on soit en mesure... d'en mesurer la propagation. Voici venu le temps des « évaluateurs », requérant de produire, à partir de prélèvements d'échantillons, une mesure de la production de l'humus des pratiques, par rapport à l'unité de surface cultivée...

Ces jaugeurs de comportements pensent comme la lettre du dictionnaire : ils ne cessent de signifier que qui dit « efficacité » postule « le maximum de résultats avec le minimum de dépense ». Dépense de quoi ? De temps et de dépense sociale. De tout ce qui retarde l'effet et rallonge l'intervalle entre l'action et son produit. Travail « à l'économie » qui induit un déni tranquille de l'acte, de la praxis comme telle. Voilà où nous en sommes.

\section{Le symptôme à l'économie}

Quand il s'agit de « traitement psychique », l'effet attendu est bien la suppression du symptôme par neutralisation.

On comprend que l'écourtement de la thérapie constitue la «philosophie » de l'évaluation. La preuve de l'efficacité de l'action serait l'obtention $\mathrm{du}$ but en un minimum temporel. Le sophisme consiste à affaiblir l'idée de fin en celle de but. Réduite à un but pragmatique, l'action peut être évaluée grossièrement, quitte à tuer sa fin dans l'œuf. L'évaluation n'a donc d'autre doctrine que le formatage du produit évaluable.

Le symptôme, promu par la psychanalyse « formation de l'inconscient 12 », se trouve à nouveau réduit à son acception simili-médicale la plus sommaire, dès lors qu'il passe sous les fourches Caudines de l'évaluation. Il n'est certes pas faux que l'acte thérapique tende à désencombrer le sujet de ses «symptômes de souffrance ». Mais le sujet a pris du temps à faire son symptôme. Formation de compromis du conflit inconscient, il est revêtu de la patine du temps, celui de la longue durée œdipienne. Ce fonctionnalisme de la « remédiation » gomme la dimension temporelle $13 \mathrm{du}$ conflit en le réduisant au délai de résiliation.

Freud a remarquablement repéré dans la volonté forcenée d'obtenir l'effet un reste de cette superstition populaire que « les névroses sont quelque chose de tout à fait superflu qui n'a absolument pas le droit d'exister 14 ». Il faut bien garder à l'esprit cette idée que cela tend à se débarrasser de cette «complication indésirée » qu'est la névrose, maladie du désir. Une thérapie bien évaluée est celle qui vient à bout de la névrose au point de confirmer qu'au fond elle était « superflue », contestation a posteriori du droit d'exister de la «subjectivité névrotique». Corrélativement, les « thérapies longues », qui s'alignent sur la temporalité de la maladie du désir, sont suspectées de complicité avec la névrose. C'est sans doute le fond de l'affaire et du contentieux.

\section{L'acte et le savoir : la magie}

L'efficacité se réfère à ce qui est le plus spécifique à l'agir, soit que «ça marche »... ou pas, que l'on comprenne... ou non. C'est là le versant magique de l'acte. L'évaluation des psychothérapies s'inscrit dans une logique de gestion, tout en promouvant une réactivation « magiste », en un style de néosuggestion.
Il y a un savoir inhérent à l'agir - ce qui est de l'ordre du « savoirfaire »- qui prend à contre-pied l'ordre du savoir même. Non que l'on puisse faire n'importe quoi, mais comment agit ce que l'on fait ? Voilà le point aveugle de l'agir, le scotome central dans le champ de sa vision. C'est en ce point que l'on trouve le registre magique de l'acte, en contraste de la science, soucieuse de produire l'intelligibilité de son processus.

Cela ne voue pas le thérapeute au statut de magicien, celui qui exploite aux fins d'illusion la magie de l'acte. Que l'acte analytique aussi touche à la magie, Freud le concède, sauf à préciser qu'il s'agit de " magie lente 15 ». Amendement humoristique : la magie justement entretient l'illusion du caractère immédiat de l'effet de son acte - ce qui fait du prestidigitateur le champion de la séance tenante. Le magicien cherche l'efficience et occulte le savoir, aux yeux de son public si ce n'est à luimême, puisque c'est du semblant que se promeut son acte. Que l'on comprenne, et le charme est rompu. Parler de magie lente, c'est dissocier la magie analytique de son aura magique, c'est y introduire une autre temporalité.

En maintenant le rapport à la magie, Freud maintient pourtant un point obscur, une « discrépance » entre savoir et acte. On ne sait pas comment « ça agit ». Après tout, les prestations des magiciens demeurent évaluables. On entrevoit même en contraste que, de méconnaître l'acte, l'évaluation s'appuie sur une certaine mentalité magique laborieusement parée des vertus de la quantification. C'est son côté comique, d'introduire du chiffre dans un acte qui lui échappe. Les thérapies dites cognitivo-comportementales culminent dans un appel « magique » à la «persuasion verbale », qui constitue le comble de la suggestion, sa forme la plus naïve. Fatalité dès lors qu'il s'agit de déposséder le sujet de la croyance à son propre symptôme, réduit à une « distorsion cognitive » et à une sorte d'apraxie.

\section{De l'évaluation \\ du but au déni de la fin}

Point de problématique de l'efficience sans dimension de finalité. Cela pose la question du rapport entre l'effet et la fin. S'ils sont liés, on se gardera de les confondre. C'est même précisément cette confusion qui, réduisant l'acte à la prestation, le soumet à la procédure d'évaluation.

Là où il y a action ou acte, il y a but. La finalité suppose une chose à réaliser. L'action s'éteint donc avec la fin qu'elle atteint. D'où l'homonymie,
La preuve

de l'efficacité

de l'action

serait

l'obtention

du but en un

minimum

temporel.

Le sophisme

consiste à

affaiblir l'idée

de fin en celle

de but.

12. P.-L. Assoun, Psychanalyse, Presses universitaires de France, 2e éd., 2007.

13. P.-L. Assoun, « Le temps de l'analyse », journée d'étude de l'École de propédeutique pour la connaissance de l'inconscient, 13 octobre 2007, publication interne de l'EPCI.

14. S. Freud, Nouvelles conférences de psychanalyse, G.W.XV, 165.

15. S. Freud, La question de l'analyse profane, G.W.XIV. 16. S. Freud, L'analyse finie et infinie.

17. P.-L. Assoun « Métiers impossibles, actes nécessaires », journée du 8 juin 2007 de l'ECART, hôpital Esquirol, «Impossible mais quand même ! » mais aussi l'équivocation entre la finbut et la fin-terme.

De plus, l'efficacité renvoie au registre pragmatique qui paradoxalement tue l'acte. Une vieille et déterminante opposition peut nous aider ici, celle, soulignée par Aristote, entre poiesis, activité définie par son effet-résultat, et praxis, action qui a sa fin en elle-même. La poeisis se définit par son impératif - conditionnel - d'atteindre son but (registre de l'efficacité) en sorte qu'elle disparaît avec l'effet atteint dans et par l'objet artificié ; tandis que la praxis s'ordonne à la finalité - inconditionnelle - de se réaliser comme acte. C'est la puissance de l'acte qui ordonne la portée et la valeur de l'effet.

Cette distinction, déterminante dans l'opposition entre technique et éthique, suggère la vraie dimension de la question. Il nous faut bien passer par cette métaphysique de l'acte pour poser en ses justes termes la question de l'efficace. L'évaluation consomme le clivage de l'acte et de la procédure : elle accomplit, dans la « mesure » de la procédure, l'oubli de l'acte.

\section{Le «paradoxe " technique : le « contre-effet »}

Pourquoi et en quoi tout cela parle-t-il à notre champ ? C'est que l'effet, « fin » de facto, de l'analyse est bien de venir à bout des symptômes. Sauf à rappeler que le symptôme n'est pas réductible à une dysfonction mais à un effet de vérité, celle du sujet divisé par le sexuel. Remémoration, répétition et perlaboration permettent de mettre au jour l'effet textuel du symptôme et d'en vider la jouissance. Le « traitement psychique », ainsi revisité par les processus inconscients, situe l'acte analytique.

Mais voici qui va planter une épine dans l'entendement évaluatif. En contraste de ce réalisme pragmatique de la remédiation, Freud situe « l'analyser » (on notera l'aspectuel) - avec le « gouverner » et « l'éduquer »-, comme l'un des trois «métiers impossibles »: «L'analyser, lit-on dans la section VII de L'analyse finie et l'analyse infinie, est le troisième de ces " métiers impossibles » dans lesquels on peut être sûr d'avance de l'effet insuffisant » (souligné par nous ${ }^{16)}$. Cela va bien au-delà de l'exclamation : "Je fais un métier impossible ! » Freud pointe ainsi, en contraste de toute action programmable, l'engagement intraitable de l'acteur dans l'impossible de son acte 17 . 
Énoncé tranquillement subversif d'une rationalité technologique d'une somme d'effets évaluables: la moindre des choses exigible d'une technè, c'est qu'à défaut de le produire immanquablement, elle s'attende à son effet, qu'elle en présume le succès - qu'il vienne ou non. Le terme Erfolg employé par Freud signifie en allemand à la fois le résultat ou l'effet (satisfaisant) et l'atteinte de ce à quoi l'on tend et la reconnaissance, bref le « succès ». De fait, le succès n'est autre que l'effet plein, donc « réussi », de l'action. Or, là, dans l'acte analytique, non seulement l'effet est in-certain, mais - on a bien lu - ce dont on peut être d'avance assuré, c'est de "l'effet insuffisant » (des ungenügenden Erfolgs)».

Cette dernière expression n'expose-t-elle pas l'analyse à un « zéro pointé » dans l'échelle de score évaluative ? En fait il n'y a pas là que réserve, mais indication de la rencontre de la puissance même du désir qui déjoue, comme à plaisir, l'intention de guérir. «Impossible », notons-le, s'applique non à l'acte, mais au « métier ». Un métier consiste à produire quotidiennement des effets suffisamment anticipables. Il y a bien un métier analytique : le " métier impossible », ce n'est pas celui qui est impossible à exercer, mais celui qui doit intégrer dans son acte la pensée structurale d'une in-suffisance de l'effet produit.

\section{La guérison à l'épreuve de la psychanalyse}

Cela fait sortir la psychanalyse de cette logique de l'évaluation, ça l'en exorbite en quelque sorte, non en la plaçant, en quelque aveu coupable, du côté d'une sorte d'absolu « inévaluable » et arbitraire, mais en contra-position d'une réduction à quelque engeenering de l'action. C'est même pourquoi «l'évaluationnisme » s'excite spécialement contre la pratique analytique qui bat en brèche l'idéologie de l'action pour poser la question de l'acte. C'est bien avec l'acte analytique qu'il veut en découdre.

On entrevoit aussi ce que cette formule freudienne, coupée de tout contexte, pourrait accréditer d'une sorte d'aveu d'inefficience de la techné analytique. « On vous l'avait bien dit : la psychanalyse, ça ne guérit pas ! C'est pourquoi l'analyste fuit le protocole d'évaluation comme la peste ! » Relativisme, voire " nihilisme thérapeutique ", qui ferait sortir l'analyse de tout souci d'efficience et la mettrait audelà de toute évaluation possible. Ce n'est pas ainsi qu'il faut l'entendre : cet « agnosticisme » de l'acte ne permet que de mieux confronter l'acte, au cœur de la réalité thérapeutique, à son propre tranchant. L'illusion de l'efficacité ne met que plus à vif la question de l'efficace. On sait que la pire invective contre l'analyse aux yeux de Freud est qu'elle «ne peut pas faire de mal ». Formule de l'impuissance : (ce) qui ne peut pas faire de mal ne fera jamais le moindre «bien ».

Dire que l'analyse est cet acte où l'on peut être sûr... de l'in-attendu n'implique pas quelque désinvolture envers l'effet. Cela ne fonde aucun indifférentisme de l'efficace ni quelque romantisme de la singularité absolue ou de l'échec magnifique. C'est même précisément le contraire. La psychanalyse ne fait pas « la fine bouche » face à l'efficience - science de l'effet -, elle n'accrédite pas de phobie de l'efficacité. Elle n'en met que plus à nu la question de l'efficience, ce qui fait entendre le fameux « ne s'autoriser que de lui-même » de l'analyste.

Nous rencontrons là la question de l'effet-limite, soit la guérison. Ce terme, emprunté à la raison médicale, vient saturer l'efficace supposé. Cela ne dissuade pas d'aller revisiter comment le créateur de la psychanalyse se définit par rapport à cet idéal régulateur de l'efficace, cela en crée même la nécessité.

\section{Du « traitement \\ psychique » \\ à « l'intraitable »}

Cela nous reconvoque à la question de la «psychothérapie ». Au fond de l'horizon de l'évaluation (psycho)thérapique est bien la guérison. L'amélioration se situerait dans la bonne moyenne, la guérison confine au « 20 sur $20 »$.

L'affaire est plus complexe : car d'un côté, « l'évaluationnisme » suspecte l'analyse de ne pas guérir, de maintenir le patient en une condition de traitement sans fin, d'ajourner la guérison sine die; d'un autre côté, il écarte et enterre l'idée même de guérison. L'essentiel est d'isoler le symptôme (au format réduit) pour le cibler - sauf à le rallonger de groupements idoines : c'est l'heure de multiplication des « syndromes », signe d'un déni des structures subjectives - et de le réduire. La métaphore du « foyer » chère aux thérapies brèves l'exprime bien : le foyer pathologique est comparé à l'usage physiologique du foyer infectieux qu'il s'agit de réduire, en laissant de côté la dynamique d'ensemble de production du symptôme. Cette « focalisation » récuse le diagnostic globalisant. Pourquoi dès lors ne pas évaluer le score de réduction du foyer infectieux ?

Freud l'avait bien vu, qui comparait le « thérapeute bref » à qui voudrait écarter la lampe d'une maison en flammes 18 . Peu importe que la maison brûle avec le sujet, peut-on ajouter, l'essentiel est l'évaluation des coûts et « l'assurance »... contre la « peine de désirer 19 ».

Cela donne l'occasion de revisiter la dimension de « guérison » que Freud maintient bel et bien à l'horizon de l'intervention analytique. Une thérapie est la rencontre de la volonté ou « souhait de guérir » du patient et de «l'intention de guérir » (Heilungsabsicht 20 ) du thérapeute. Analyste et analysant ne forment-ils pas une « foule à deux 21 »?

La question de la guérison est repositionnée à travers la «psychothérapie » ou « traitement psychique ». Celui-ci suppose qu'un «mal psychique » peut se soigner au moyen d'un procédé de guérison luimême «psychique »-ce qui suppose un effet guérisseur du processus. Idée qui, ne l'oublions pas, subvertit le préjugé médical qu'il n'est d'efficace que physiologique. La condition du côté du sujet est «l'attente croyante » (gläubige Erwartung), organe de la « volonté de guérison » (Genesungswille). On retrouve là la question de

\section{L'illusion de l'efficacité}

ne met que

plus à vif

\section{la question}

\section{de l'efficace.}

18. S. Freud, L'analyse finie et infinie.

19. P.-L. Assoun, L'entendement freudien. Logos et Ananké, Gallimard, 1984. 20. S. Freud, Chemins de la thérapie psychanalytique, G.W.XII, 188.

21. S. Freud, Psychologie collective et analyse du moi. 22. S. Freud, Sur la psychothérapie, G.W.V. 23. P.-L. Assoun, Leçons psychanalytiques sur le transfert, Economica/Anthropos, 2006 ; 2e éd., 2007. l'hypnose. Celle-ci est la démonstration expérimentale de "l'influencement psychique », c'est-à-dire de la « suggestion ». C'est à ce titre que le terme « guérison » apparaît dans le titre du tout premier écrit « psychologique » de Freud, en 1887 : «Un cas de guérison hypnotique ». Le médecin-hypnotiseur est mis en position de Wundermann, d' « homme-miracle », ce qui repositionne la question des « guérisons miraculeuses » (Wunderheilungen). Elle fait sortir la notion, essentielle dans les religions, de son aspect magique ou spirituel pour la reporter du côté de « l'influencement psychique ». Quant à la technique d'« abréaction », elle mise bien sur l'efficace de liquidation du trauma, via les réminiscences - sauf à remarquer déjà qu'en contraste de l'« épuration » de son homologue aristotélicien, la catharsis breuérienne revient à un « ramonage ». On en retrouve la forme la plus fruste dans les TCC, malgré leur « relookage » cognitif.

Point décisif : le passage de la suggestion à l'analyse, résumé dans l'écrit de 1904 « Sur la psychothérapie » : « Je désespérais de rendre les effets de la suggestion assez efficaces et assez durables pour amener une guérison définitive $22 »$ (souligné par nous). Le passage à l'analyse d'une part prend acte de l'échec de la guérison par suggestion, mais d'autre part rompt avec l'idéal de guérison inhérent à la suggestion - à laquelle ramène fatalement la conception d'une action évaluable. Là intervient la différence fameuse entre « la peinture qui opère per via di porre (en ajoutant une substance sur une toile blanche) » et « la sculpture qui opère per via di levare en enlevant à la pierre brute tout ce qui recouvre la statue qu'elle contient ». La psychanalyse, prenant en compte la résistance, ne tend pas à accomplir la guérison par adjonction mais à dégager, de dessous l'enveloppe résistante, la « statue »- sauf à « lever» et vider la jouissance du symptôme. À l'idée d'efficacité par complémentation, s'oppose la notion d'efficace par décomplémentation...

\section{Transfert et \\ vœu de guérison}

Mais voici un élément qui va décider de la position proprement psychanalytique repositionnant la problématique de l'efficace : soit l'effet, au-delà de la résistance, du transfert.

De cette immense question, déterminante de l'acte analytique, traitée ailleurs 23 , pointons l'incidence paradoxale sur la logique de la guérison. Le transfert fournit le ressort détermi- 
nant : il cristallise le « désir de guérir » en instituant la « névrose de transfert », ce « royaume intermédiaire entre la maladie et la vie $24 »$.

On comprend que la réduction du symptôme à un dysfonctionnement et de la thérapie à une remédiation traduit le déni de ce «moteur » de l'acte qu'est le transfert, soit cet événement qui surgit comme modalité de l'amour inconscient, au cœur de l'acte. C'est la figure du transfert comme rencontre foncièrement in-attendue (alors même que l'on s'y attend) qui réalise l'irréductibilité de l'acte au dispositif de l'action. Le transfert constitue en ce sens la « démesure » de l'action thérapeutique. La « raison évaluative »a pour effet et finalité de l'évacuer.

Il apparaît que c'est la haine $d u$ transfert qui est au principe de l'évaluationnisme. Dans le paramétrage de l'action, ce qui est chroniquement et principiellement exclu, c'est précisément le transfert, soit le catalyseur de l'acte. C'est aussi la dimension folle de l'évaluation, celle d'épurer l'action de tout son réel transférentiel, le réduisant à une coquille vide. C'est son abstraction fondamentale. C'est aussi ce qui ramène la relation thérapeutique aux formes de suggestion les plus élémentaires.

\section{Du danger de guérir au refus de guérison}

Voici alors l' « os » par lequel la psychanalyse va rompre avec toute thaumaturgie ou sacralisation de l'effet et exposer l'action à une grave dé-valuation. «L'efforcement thérapeutique », naviguant entre rendre conscient le ça et corriger le moi, se heurte au retour des « résistances contre la guérison » : « Il en résulte que la guérison même est traitée par le moi comme un nouveau danger 25. » « Il n'est pas d'impression plus puissante des résistances pendant le travail analytique que celle d'une force qui se défend avec tous ses moyens contre la guérison (Genesung) et peut se maintenir tout à fait dans la maladie et la souffrance 26 . » Tel est le « sentiment de culpabilité » ou « besoin de punition » qui se joue entre le moi et le surmoi. Dimension masochiste que l'acte analytique met au jour en butant dessus et du fait même de s'y exposer 27 .

L'annonce d'amélioration ou promesse de guérison provoque régulièrement la « réaction thérapeutique négative », comme si le sujet contrait l'efficace à partir de son annonce (erreur qui peut servir de leçon aux thérapeutes naïfs). C'est le problème de la Verschlimmerung, le fait d'aller plus mal en cours de cure, mentionné pour la première fois dans Remémora-

\section{Il apparaît que}

\section{c'est la haine}

du transfert

qui est au

principe de

\section{l'évaluation-}

\section{nisme.}

24. S. Freud, Remémoration, répétition et perlaboration, G.W.X, 135

25. S. Freud, L'analyse finie et infinie, sect. V, G.W.XVI, 84. 26. Op. cit., sect. VI, G.W.XVI, 88.

27. P.-L. Assoun, Leçons psychanalytiques sur le masochisme,

Anthropos/Economica, 2003 ; 2e éd., 2007.

28. S. Freud, Le moi et le ça, ch.V, G.W.XIII, 278-279. 29. S. Freud, « Ceux qui échouent devant le succès », dans Quelques types de caractères tirés $d u$ travail analytique et notre commentaire, P.-L.Assoun, Le préjudice et l'idéal. Pour une clinique sociale du trauma, Economica, 1999.

30. S. Freud, Inhibition, symptôm et angoisse, ch. XI, G.W.XIV, 193.

31. S. Freud, Nouvelles conférences d'introduction à la psychanalyse, G.W.XV, 117. 32. S. Freud, L'analyse finie et infinie, sect.VI, G.W.XVI, 88 33. S. Freud, Le problème économique du masochisme, G.W.XIII, 378 tion, répétition, perlaboration, puis dans le cas de l'Homme aux loups, mais développée le plus explicitement dans Le moi et le ça : «Quand on... donne espoir (à ces patients) et que l'on exprime sa satisfaction sur l'état du traitement, ils semblent insatisfaits et aggravent régulièrement leur état... On se convainc que ces personnes ne supportent pas l'éloge ni la reconnaissance, mais qu'ils réagissent aux progrès de la cure de façon fausse. Chaque solution partielle, qui devrait avoir pour conséquence une amélioration ou une disparition temporaire des symptômes chez les autres, éveille chez eux un renforcement momentané de leur souffrance, ils vont plus mal (sich verschlimmern) pendant le traitement au lieu d'aller mieux. Ils montrent une réaction thérapeutique négative ${ }^{28}$. » Conclusion : " Pas de doute que quelque chose en eux s'oppose à la guérison dont l'approche est redoutée comme un danger. On dit que chez ces personnes, ce n'est pas la volonté de guérir (Genesungswille) mais le besoin de punition qui prend le dessus ». Force antagoniste du « désir de guérison ».

Cet « échec devant le succès » (Scheitern am Erfolg 29) est ce qui vient, tel le ver dans le fruit, contrarier l'efficace. Dans Inhibition, symptôme et angoisse, il est mentionné comme forme de résistance émanant du surmoi : « il s'oppose à tout succès et par suite aussi à la guérison par l'analyse 30 ». Confirmation dans la XXXIIe des Nouvelles conférences : quelques «mots d'espoir» sur les progrès de l'analyse « suffisent à provoquer une aggravation de l'état »: «Le nonanalyste dirait qu'il manque du « vouloir de guérison » («Genesungswillens »). Le « mode de penser analytique voit « dans ce comportement une expression du sentiment de faute inconscient, l'être malade avec ses souffrances et empêchements $31 »$. La résistance thérapeutique négativante apparaît comme une arme contre « la prestation d'aide analytique »- ce qui se rebaptise « masochisme immanent 32 » : «La satisfaction de ce sentiment de culpabilité inconscient est peut-être le poste le plus puissant du bénéfice de maladie composé en général de la somme de forces qui se cabre contre la guérison (Genesung) et ne veut pas abandonner l'être malade. » La résistance thérapeutique négativante est en ce sens « l'une des résistances les plus lourdes et le plus grand danger pour le succès de nos intentions thérapeutiques ou éducatives 33 ».

Épine plantée dans la rationalité de l'efficacité. De toute cette dimension, il ressort que c'est en somme le sujet qui travaille à dévaluer l'action thérapeutique... C'est le retour de la pulsion 
de mort dans le processus qui y inscrit cette déliaison, élément « sabordeur » du processus qui voue du même coup à la dérision l'arithmétique de l'évaluation, si éloignée du réel du symptôme.

\section{Le désir de guérison et sa temporalité}

Mesurer la puissance de ce que l'on peut tenir pour un « contre-effet » ne débouche pas sur quelque pessimisme thérapeutique unilatéral. Freud suggère dans Chemins de la thérapie analytique une dynamique de la guérison très précieuse, à méditer pour saisir les enjeux de la temporalité de l'efficace : « Vous pouvez observer pendant la cure que chaque amélioration de son état de souffrance retarde le rythme de rétablissement (das Tempo der Herstellung) et restreint la puissance pulsionnelle qui pousse à la guérison 34 . » Laisser ouverte jusqu'au bout la pulsion de guérison, voilà qui fonde la temporalité analytique. L'effet de l'acte dès lors se mesure-t-il ? On trouve dans le cas de l'Homme aux loups une sorte d'éloge de la durée : Freud attire l'attention sur le fait que « si les analyses aboutissant en peu de temps à une issue favorable sont pour le thérapeute une source de vanité (Selbstgefühl), elles restent la plupart du temps sans intérêt pour la recherche scientifique $35 »$. En effet : « On n'apprend rien de neuf par elles. Si elles réussissent si rapidement, c'est parce que l'on savait déjà tout ce qui était nécessaire pour leur traitement. C'est là où il y a une difficulté que l'on pénètre dans les couches les plus profondes et les plus primitives du développement psychique. »

C'est alors que l'analyse mérite son nom. C'est dans l'introduction du cas le plus long, il est vrai, de sa pratique que Freud formule une réponse à l'éloge du bref.

Le raccourcissement de l'acte, comme le montre la pathologie de l'acte sexuel, n'est pas un bon signe du désir. Pour le dire brutalement, les théories courtes s'engagent dans une logique de l'éjaculation précoce... du symptôme. Ce que l'évaluation institue en idéal normatif du « vite fait, bien fait » non fait..

\section{Le malaise évalué}

Nous étions partis du constat freudien de cet effet de tromperie anthropologique dans l'évaluation. La naïveté anthropologique de l'évaluationnisme s'appuie sur l'idée que l'homme est capable d'évaluer l'homme, en une sorte d' « anthropométrie », accomplissant le déni du rapport divisé du sujet à sa vérité.

Mais précisément, la réglementation par l'évaluation n'atteint la psy-

\section{Nul doute que}

\section{Bouvard et}

\section{Pécuchet,}

\section{de retour,}

chercheraient

\section{des livres sur}

\section{l'évaluation.}

34. S. Freud, Chemins de la thérapie analytique.

35. S. Freud, À partir d'un cas de névrose infantile.

36. P.-L. Assoun, Freud et les sciences sociales. Psychanalyse et théorie de la culture, Paris, Armand Colin, 1993 ; rééd. 2008 37. P.-L. Assoun, « L'ignorance passionnée. Bouvard et Pécuchet saisis par la psychanalyse », dan Analyses et réflexions sur Gustave Flaubert : Bouvard et Pécuchet, Ellipses/Marketing, 1999 .

p. 104-115

38. P.-L.Assoun, « La recherche freudienne. Petit Discours de la méthode à l'usage de la Recherche en psychanalyse », dans « La recherche en psychanalyse à l'Université », Recherches en psychanalyse, 2004-1, L'Esprit du temps, p. 49-63.

39. S. Freud, Au-delà du principe de plaisir, ch.VI, G.W.XIII, 64 40. S. Freud, op. cit., p. 65.

41. P.-L. Assoun, Introduction à lu métapsychologie freudienne,

Paris, PUF, 1993, p. 62 ;

L'entendement freudien, op. cit. chothérapie qu'au bout d'un trajet qui affecte l'idéal même du savoir selon les normes sociales, ce qui donne une photographie du «malaise de la culture » actuel, sur fond du malaise structural de la culture 36 .

Cette rage d'évaluer, au-delà de la défense de la norme sociale, renvoie à une angoisse sourde particulière. On s'avise bien vite que, loin de surenchérir sur le savoir, elle se présente comme une réponse, à la fois obstinée et affolée, à un terrible vide central de savoir. Nul doute que Bouvard et Pécuchet 37 , de retour, chercheraient des livres sur l'évaluation, sauf à ne trouver que des modes d'emploi et des notices d'utilisation.

Le protocole d'évaluation s'impose comme la forme (post)moderne du discours du maître. Plus question de maître qui prescrit, mais il s'écrit dans l'énonciation : «Évaluons-nous / Évaluez-vous » dont la conclusion logique est : «Évaluons... vous ».

Il y a plus précis. Dans un monde social où le savoir est dissocié de la vérité, où nul ne « peut dire », la référence à l'expertise intervient comme bouche-trou du clivage qui s'en aggrave d'autant.

La machine, coupure épistémologique déterminante, a pris la figure de régulation sociale de la pensée, « jouissance réglementée ».

\section{Le surmoi auto-évaluateur}

L' « auto-évaluation », la vraie, n'est pas épargnée au chercheur, elle est déterminante. Le chercheur 38 , c'est celui qui, entre deux évaluations institutionnelles (de plus en plus rapprochées), se confronte au pire évaluateur, le plus exigeant, soit son propre surmoi. Ce que le chercheur en psychanalyse exprime très précisément : en s'approchant des « choses dernières, des grands problèmes de la science et de la vie », il bute sur une limite subjective, soit le fait que « chacun est dominé par des préférences profondément enracinées » qui sous-tendent son travail de spéculation ${ }^{39}$. Suit la formule de l'autoévaluation : il ne lui reste, une fois qu'il s'est affecté de la défiance salubre envers lui-même, à se régler sur « une bienveillance froide pour les produits de son propre efforcement de pensée 40 ».

Freud donne là la formule du " surmoi métapsychologique 41 », qui renvoie dos à dos le subjectivisme et l'objectivisme illusoire. La surenchère de l'évaluation pourrait bien consister à narcotiser le surmoi en le renvoyant à l'anonymité d'un protocole. 